\title{
PROSPECTS OF BRAIN-MACHINE INTERFACES FOR SPACE SYSTEM CONTROL
}

\author{
Carlo Menon ${ }^{1}$, Cristina de Negueruela ${ }^{1}$, José del R. Millán ${ }^{2}$, Oliver Tonet ${ }^{3}$, Federico \\ Carpi $^{4}$, Michael Broschart ${ }^{1}$, Pierre Ferrez ${ }^{2}$, Anna Buttfield ${ }^{2}$, Paolo Dario ${ }^{3}$, Luca Citi ${ }^{3}$, \\ Cecilia Laschi ${ }^{3}$, Mario Tombini ${ }^{5}$, Francisco Sepulveda ${ }^{6}$, Riccardo Poli ${ }^{6}$, Ramaswamy \\ Palaniappan $^{6}$, Franca Tecchio ${ }^{7}$, Paolo Maria Rossini ${ }^{5}$, Danilo De Rossi ${ }^{4}$ \\ ${ }^{1}$ Advanced Concepts Team, European Space Agency, ESTEC, Noordwijk, The Netherlands \\ ${ }^{2}$ IDIAP Research Institute, Martigny, Switzerland \\ ${ }^{3}$ CRIM Lab, Scuola Superiore Sant'Anna, Pisa, Italy \\ ${ }^{4}$ University of Pisa, Interdepartmental Research Center “E. Piaggio”, Pisa, Italy \\ ${ }^{5}$ Neurology Department, Università Campus Biomedico, Rome, Italy \\ ${ }^{6}$ BCI Group, Department of Computer Science, University of Essex, Colchester, UK \\ ${ }^{7}$ Istituto di Scienze e Tecnologie della Cognizione (ISTC), CNR, Rome, Italy
}

\begin{abstract}
The dream of controlling and guiding computer-based systems using human brain signals has slowly but steadily become a reality. The available technology allows real-time implementation of systems that measure neuronal activity, convert their signals, and translate their output for the purpose of controlling mechanical and electronic systems. This paper describes the state of the art of non-invasive Brain-Machine Interfaces (BMIs) and critically investigates both the current technological limits and the future potential that BMIs have for space applications. We present an assessment of the advantages that BMIs can provide and justify the preferred candidate concepts for space applications together with a vision of future directions for their implementation.
\end{abstract}

\section{INTRODUCTION}

Brains are characterised by every property that engineers and computer scientists detest and avoid. They are incredibly complex, and their processes at every level are chaotic, unstable, nonlinear, non-stationary, non-Gaussian, asynchronous, noisy and unpredictable. Yet brains, and human brains in particular, are undeniably among the most successful "devices" that evolution has produced.

There is a growing interest in the use of brain signals for communication and operation of devices, in particular for physically disabled people. Brain states can be detected and translated into actions such as selecting a letter from a virtual keyboard, playing a video game, or moving a robot arm. Such devices, which do not require the user to perform any physical action other than thinking, are called brain-computer interfaces (BCI) or brain-machine interfaces (BMI).

While communication between humans has been extensively developed and studied, communication between people 
and devices - especially sophisticated electronic systems - is at a relatively primitive stage. Only 60 years ago, state of the art computing systems like ENIAC required punch cards for communication. Modern means of interfacing with a computer such as a keyboard and mouse are vastly superior, but remain unintuitive and unnatural.

For decades scientists have speculated on the possibility of a direct interface between a brain and a machine. In the 1970s the basic ideas were put forward and initial experiments based on the analysis of brain electrical activity generated in response to changes in gaze direction were successfully carried out. In recent years a variety of prototypes based on these and other principles have been developed.

The possibility of controlling external devices using BMI technology could have a tremendous influence on strategic plans for future space missions. For this reason, we have critically investigated existing BMIs in order to assess the maturity of this technology and estimate the improvements that it could yield [1-3].

The paper is organised as follows. We first describe the fundamental principles on which BMIs are based (Section 1). Section 2 presents the case study of BMIs based on electroencephalographic signals, while Section 3 discusses other possible BMI technologies. In Section 4 we review applications currently being developed for BMIs. Sections 5 and 6 investigate how space activities could benefit from the use of BMIs, and Section 7 analyses the effect of the harsh space environment on possible future use of BMIs. Section 8 critically discusses the current challenges that researchers are facing in order to obtain reliable BMIs and Section 9 presents some future prospects for the development of BMIs.

\section{BRAIN-MACHINE INTERFACES}

BMIs can be defined as any system that can monitor brain activity and, from it alone, translate a person's intentions into commands to a device. A BMI transforms bioelectrical brain signals (and other types of signals such optic reflection, etc), modulated by mental activity (e.g. imagination of hand movement) into control signals.

There are a variety of methods that have been employed to monitor brain signals. They include, but are not limited to: electroencephalographic signals (EEG), magnetoencephalographic signals (MEG), positron emission tomography (PET), functional magnetic resonance imaging (fMRI), optical imaging (NIRS, nearinfrared systems) and implanted means for recording electrical activity, e.g., via electrocorticograms. Among these methods, EEG seems to have the two properties that could lead to the implementation of BMIs for future space mission: non-invasiveness and portability.

However, regardless of the sensor type, there are a number of elements that are common to all BMIs (Figure 1). As a first step, an experimental protocol must be designed depending on the specific application. This includes the choice of mental task and stimulus parameters (e.g., visual scenery timing and constraints), as well as the minimisation of unwanted stimuli and distractions that may affect the properties of the signals to be monitored. Once a suitable protocol is designed, signals can be monitored and acquired with the precise instrumentation. The signals are then converted to digital format and filtered and used for the extraction of information relevant to the mental task under investigation. Before the signals are used, however, some unwanted artefacts may need to be 
removed (e.g. large amplitude oculomotor signals).

Once any unwanted signal components are attenuated as much as possible, features can be extracted from the data and fed into a translation algorithm, which can be as simple as 'if-then' rules or a previously determined linear classifier. However, it is quite often the case that the complexity of the data requires the use of non-linear classifiers and machine learning algorithms. The translation algorithm then yields commands to be used by various devices (the 'control' case) or to lead to explicit communication of thoughts (the 'communication' case).

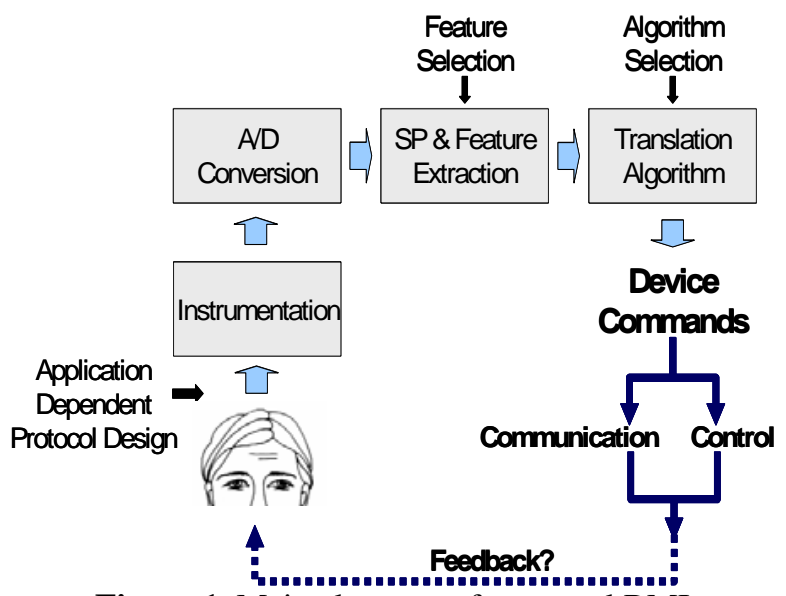

Figure 1. Main elements of a general BMI

The different methods of monitoring brain activity can be coarsely classified as invasive and non-invasive. Invasive methods make use of implanted electrodes (e.g. subdural, epidural or intracortical electrocorticogram) while non-invasive methods use electrodes (or other sensors) applied to the skin surface.

So far, the invasive monitoring has been found to be the most effective. Outstanding results have been achieved with implanted devices where primates are able to guide robotic manipulators by means of their neural activity with spatial errors in the order of centimetres [4].
Recently, a human being has used an invasive BMI as well [5]

The non-invasive monitoring is, to date, less efficient. However, this could have a more direct impact for society and space applications because of its reduced risk, more favourable public perception, easy donning and doffing, and shorter time frame for qualification in human beings.

\section{NON-INVASIVE BMI:} THE CASE OF EEG

The most commonly studied potential interface for humans has been electroencephalography (EEG), mainly due to its fine temporal resolution, ease of use, portability, and cost of set-up. However, practical use of EEG as a BMI requires considerable user training and is highly susceptible to noise.

Recent studies have shown that imagining the execution of a particular sensory-motor task gives rise to almost the same pattern of neuronal activity in Central Nervous System as actual performance of the sensory-motor task [6]. The current state-of-the-art permits the correct decoding of EEG signals to a very large extent. The main problem in these approaches is that single trial EEG data is very noisy.

\section{$2.1 \quad$ Signals}

EEG measures the electrical activity by detecting the electrical potential difference between a point on the scalp and ground. The tissues between the electrode and the monitored cells, and the distance between the neurons and the electrodes, cause the signal to be attenuated by many orders of magnitude. Thus, the activity of a few neural cells cannot be detected through EEG. Instead, EEG is a result of joint activity of 
thousands of underlying neurons activated together

For certain stimuli, such as flashed images and lights, the EEG exhibits a strong characteristic signal, the so-called evoked potential, which reflects the immediate automatic responses of the brain to those external stimuli. While evoked potentials are easy to pick up with electrodes and have been used in the context of BMIs, the necessity of external stimulation restricts their applicability. A more natural and suitable alternative is to analyse components associated with either of two kinds of spontaneous "intentional" mental activity: slow potential shifts or variations of rhythmic activity.

\section{$2.2 \quad$ Training}

Some subjects can learn to control their brain activity through appropriate - but lengthy - training in order to generate fixed EEG patterns that the BMI transforms into external actions. An alternative is to use machine-learning approaches to train the classifier embedded in the BMI. Most of these approaches are based on a mutual learning process where the user and the brain interface are coupled together and adapt to each other. This should accelerate the training time.

Typical tasks intended for subject training include positioning a cursor, tracking a moving object, or selecting a target. Once these skills are acquired, the subject can progress to applications that perform real-world tasks such as communication, controlling the environment, or moving robotic limbs.

\subsection{Synchronous vs. asynchronous}

EEG-based BMIs are limited by a low channel capacity, with most of the current systems having a channel capacity below $0.5 \mathrm{bits} / \mathrm{s}$ [7]. One of the main reasons for such a low bandwidth is that they are based on synchronous protocols. These facilitate EEG analysis since the starting time of mental states are precisely known. However, EEG-based BMIs are slow and normally recognise only up to four mental states independently of the number of electrodes used to acquire the measurement.

Other BMIs use more flexible asynchronous protocols where the subject can voluntarily change the mental tasks being executed at any moment without waiting for external cues [8]. The time of response of an asynchronous BMI can be below 1 second, although their accuracy is lower than for synchronous BMIs at the moment.

\section{$2.4 \quad$ Hardware}

EEG signals are acquired with a portable acquisition system such as the one shown in Figure 2. Subjects wear a commercial EEG cap with integrated scalp electrodes that cover the whole scalp and are located according to the $10 / 20$ international system or extensions of this system to allow recordings from more than 20 electrodes (i.e., the 10/10 system)..

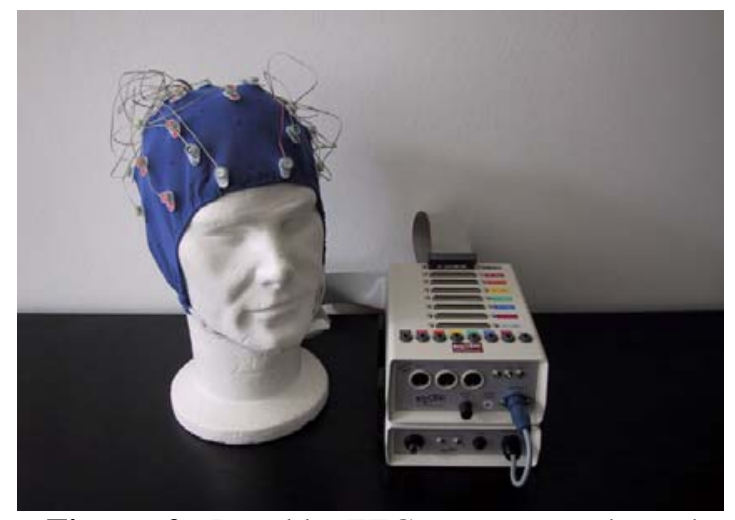

Figure 2. Portable EEG system used at the IDIAP Research Institute. It is a commercial BioSemi ActiveTwo system 
Bipolar or unipolar electrodes can be used in the EEG measurement. In the first method the potential difference between a pair of electrodes is measured, while in the latter the potential of each electrode is compared either to a neutral electrode (placed, e.g., somewhere on the body) or to the average of all electrodes.

EEG electrodes may bring many practical problems, like sensitivity to electromagnetic radiation, difficulty to place and position, varying conductance, usually a limited number of channels, and discomfort when used for a longer time.

New trends in the design of EEG measurement systems include miniaturization, battery-powered frontends close to patient, fiber optic data transfer to the PC in charge of signal processing, and use of active electrodes. These have the property that the first amplifier stage is integrated within the electrode.

\section{OTHER NON-INVASIVE BMIs}

Present EEG-based BMIs are characterised by limited spatial resolution since the activity of individual neurons cannot be detected. Localised measurements, obtained using alternative brain measurement systems, could greatly improve performance of non-invasive BMIs. Alternative solutions for mid-term applications are PET, fMRI, NIRS, and, MEG.

\subsection{PET}

Positron Emission Tomography (PET) is based on injecting the subject a contrast medium (radionuclides of short half-life) to image a task-specific metabolic activity, i.e. cerebral blood flow and related oxidative metabolism. A scanner and a cyclotron to generate the radionuclides are needed. It is in the eye of the individual beholder, whether this technique can be classified as noninvasive or not. No real surgical brain invasion is done, but substances are temporarily injected into the patient. The main drawbacks of this technology for future space applications are the mass and size of the cyclotron and the considerable power consumption of the system. More importantly, PET requires administering radioactive substances to the user.

\section{$3.2 \quad$ fMRI}

A more suitable alternative might probably be offered by functional Magnetic Resonance Imaging (fMRI), which does not require any kind of physical invasiveness. A reasonably high spatial resolution is achieved by measuring activity-related, local haemodynamic changes (blood oxygenation level and volume) and applying a magnetic field to the subject's skull. Due to their different magnetic properties, the changing levels of hemoglobin oxygenation generate variations in image intensity. The bulkiness of the equipment is a limiting factor for real applications, but there is ongoing work on portable systems.

\section{$3.3 \quad \underline{\text { NIRS }}$}

Both PET and fMRI provide metabolic feedback as indirect responses of neuronal activity. In contrast NearInfrared Systems (NIRS) penetrate very deeply into the cerebral tissue, providing both a haemodynamic and a neural response related to activity. The first one is relatively easy to acquire, whereas the second response is very noisy. NIRS hardware is lightweight and portable, in principle consisting of a pair of light emitting fibre optics and receivers and a 
small unit to be strapped closely to the head. Incident radiation results in spectral refraction, diffuse reflectance, or absorption. As opposed to fMRI, which requires the subject to keep a defined spatial position, NIRS allows the subject a good mobility as the sensor-detector unit can be easily fixed on the skull.

\subsection{MEG}

Magnetoencephalography (MEG) is based on the measurement and the analysis of magnetic fields generated outside the scalp by brain activity, derived either from excitatory or inhibitory post-synaptic potentials. Although there is no distortion by tissue inhomogenities between the emitters and sensors, the detection of the weak magnetic signals is extremely challenging. Ultra sensitive magnetic field detectors and precise noise cancellation techniques are required to limit the effects of strong background noise. The main advantage over EEG is that brain signals of better quality can be more locally detected. This might improve the learning process of the user. However, from an applicative point of view, modern MEG systems are stationary, require adequate shielding, and do not allow mobile use for astronauts.

\section{CURRENT APPLICATIONS}

BMI systems are being developed to operate a number of brain-actuated applications that augment people's communication capabilities, provide new forms of entertainment, and also enable the operation of physical devices. There exist, for example, virtual keyboards for selecting letters from a computer screen and write a message. Of these systems, the evoked BCI [9] has the highest performance, but as mentioned before, the need for external stimulation makes it less natural to use. Using the alternative spontaneous BCI systems, subjects can write a letter in times ranging from 22 seconds to 2 minutes [10, 11].

Several "brain games" have been developed by Millán's team in Switzerland, Pfurtscheller's team in Austria and Müller's team in Germany. In the first case, the "brain game" is the classical Pacman [10]. In order to control Pacman, two mental tasks are sufficient. These are used to make Pacman turn left or right, a change in direction of movement whenever one of the mental tasks is recognized twice in a row.

The main application for which BMIs are currently under research is to assist disabled people, for example, to restore functionality in patients who lost the ability to move their limbs (tetraplegia).

\section{BMIs FOR SPACE} APPLICATIONS

The space environment is inherently hostile and dangerous for astronauts. For this reason, Extra-Vehicular Activity (EVA) should be limited as much as possible and robotic systems should be used instead. It would be desirable to optimise the interface between astronauts and external semi-automatic manipulators. In the event of an EVA, BMIs can also prove useful for environmental or external control since astronauts have limited mobility for operation inside their space suits.

The theoretical advantages of using BMIs are numerous, for example, commands could be sent with minimum output delays and with high accuracy. BMI could also enable new operations leading to a new approach to mission and 
spacecraft design. Multi-teleoperations could simultaneously be performed using one single brain-machine interface. This would maximise the efficiency of astronaut activity, which is of primary concern. Alternatively, one could envisage more than one astronaut simultaneously commanding a machine during Intra- or Extra-Vehicular Activity. Robotic aids could also be useful to astronauts weakened after many months in micro-gravity.

We believe that BMIs would be of particular interest for space applications when conceived as an augmenting interface, i.e. allowing astronauts to perform actions in addition to what they already can do with their normal abilities.

Recent studies [8] suggest that there is the need to incorporate shared autonomy principles into BCIs in order to improve their performance. In shared control, the intelligent controller relieves the human from low level tasks without sacrificing the cognitive superiority and adaptability of human beings, which are necessary for decision-making and acting in unforeseen situations. In other words, in shared control there are two intelligent agents, the human user and the robot. The user only conveys his intents to the robot that then executes them autonomously. [12]

Regarding the possible future introduction of BMI technologies into spaceflight, we believe that this should be done to complement existing technologies, not to replace them. For example, a first step could be the control of secondary and non-critical onboard computers. Only demonstrating the performance of BMIs in non-critical situation could we envisage their adoption for more critical operations.

\section{PREFERRED CANDIDATE CONCEPTS}

In order for BMIs to be advantageous systems for short-term space applications, they need to be characterised by certain fundamental properties:

Non-invasiveness, because of their reduced risk and short time frame for qualification in space environment. In addition, there is a more favourable public perception of non-invasive BMIs than invasive ones.

High reliability, since there is no possibility of repair once in space.

High efficiency, to justify its benefits over other alternatives.

High sensitivity, as above.

Ease of use by the astronaut, especially taking into account the difficulty of operating in a micro-gravity environment.

Sufficient comfort for the astronaut, since functioning space is tiring and astronauts have to perform demanding tasks.

Electromagnetic compatibility with electronic equipment of the spacecraft cabin, to avoid interferences.

Low weight and volume of the driving and reading equipment, both for an affordable access to space and ease of use.

Robustness both in the interface with the body and in the response of the system under various environmental and movement conditions

The need of respecting at least all these characteristics limits the types of BMI potentially useful. In particular, it tends to 
exclude, in principle, fMRI-based interfaces and MEG-based interfaces, for the following reasons:

- both of these types require bulky and heavy equipment;

- both of these types are definitely not practical for astronauts, especially in consideration of the activities that they have to perform continuously.

On the contrary, EEG-based interfaces seem to represent the most suitable candidates for short-term applications.

In addition to BMIs, we believe that the concurrent use of additional and auxiliary human-machine interfaces could bring considerable benefits when advanced human-machine interfaces will firstly be introduced. They could in fact increase the speed and efficiency of some operations despite current BMI drawbacks. As an example of noninvasive human-machine interfaces to be used as auxiliary systems for a BMI, electromyographic signals detected from arm muscles of an astronaut may permit the control of a robotic arm. Electrical activity recorded in proximity of muscles could be elaborated and used as the control input for the robotic mechanism. Such an action may be performed, in an early stage, in parallel to those controlled by the brain interface. Therefore, the two interfaces could work at the same time to enable easier and efficient implementations of multiple tasks.

We believe that this combination of BMIs and other human-machine interfaces could ease the introduction of BMIs into space. However, in the long term we envisage that BMIs will be robust enough to be independent.

\section{ENVIRONMENT EFFECTS}

The space environment poses many hazards to living organisms, from extreme temperatures, vacuum and radiation to the effects of spaceflight and extended exposure to microgravity on the human body. In the following paragraphs, the critical effects of the environment on a correct operation of BMIs are discussed.

\subsection{Human physiology in space}

Human anatomy and physiology are the result of the evolution through time to adapt to life on Earth. Space is a completely different environment and the human body undergoes important modifications when in space, noticeable even during short journeys [13].

In addition to changes in the cardiovascular system, muscles and bone mass, the absence of gravity affects the way the sensory and balance centres within our brains perceive the outer environment and the position and the orientation of the body within it. These changes, further explained in the next section, make necessary a validation of BMIs as conceived in the $1 \mathrm{~g}$ environment of the Earth.

\subsection{Effects of microgravity on the brain}

In the virtual absence of gravity the brain must re-adapt internal models of the laws of dynamics. Gravity dictates the laws of motion of our body and limbs, as well as of the objects in the external world with which we interact. In the absence of gravity the brain has to readapt these models to the new environment and change the strategies it uses for spatial orientation.

All these changes in what the brain receives and how it processes the information can lead to modifications that can reflect on the functioning of a BMI. The signals and patterns a BMI is based upon can be disrupted, reduced, enhanced 
or changed by the different environment and its consequences. Therefore, a BMI developed and tested on Earth might stop working in space or might need a retuning of the parameters of the algorithm to adapt to the psycho-physiological modifications of the Central Nervous System.

\subsection{Effects of radiation}

Weightlessness is not the only factor affecting the human body in space. The absence of the protection of the atmosphere exposes astronauts to a steady flux of cosmic particles. Long permanence in orbit results in a dose of radiation many times that of the same period spent on Earth.

Cosmic-ray particles also interact with our visual system and give rise to the phenomenon known as a "light flash", consisting on unusual visual phenomena caused by the interaction of energetic cosmic-ray particles impinging on the retina [14]. We cannot a priori exclude that these phenomena could interfere with the operating of a Brain-Machine Interface. They could in principle act as external stimuli and trigger the activation of specific areas. Fortunately, they are quite rare and, so, their impact could be limited.

\section{CHALLENGES FOR BMIS}

To be effective in real-world applications, many challenges inherent to employing BMI control must be addressed and overcome. These challenges are common to both space and rehabilitation applications and can be generalised in several categories [15] as presented in the following paragraphs.

\subsection{Throughput}

Even the best average information transfer rates for experienced subjects and well-tuned BCI systems are relatively low, in the vicinity of $24 \mathrm{~b} / \mathrm{min}$ (roughly three characters per minute) [7]. This is too slow for natural interactive communication. So, in order to effectively use BMIs as an alternative to conventional interfaces, it is necessary to research ways of optimising selection techniques and incorporating prediction mechanisms to speed up communication.

\subsection{High error rate}

A significant complicating factor in the slow information transfer rate of BMI users is the high probability of errors. Brain signals are highly variable, and this problem is exacerbated in severely disabled users by fatigue, medications, brain remapping due disability, and some medical conditions. Devising methods of quickly resolving or preventing errors is critical to successful BCI interaction.

A way of preventing the execution of erroneous commands is to detect directly the users' EEG potentials generated in response to errors made by the BCI [16].

\section{3 $\underline{\text { Autonomy }}$}

Ideally, a communication system for a person with severe disabilities should be completely controlled by its user. Unfortunately BMI systems require extensive assistance from caretakers who need to apply electrodes or signalreceiving devices before a user can communicate.

Also in the case of space operations, where astronauts are operating in difficult conditions, like in micro-gravity, the setup and initiating procedures should be made easy. 


\subsection{Cognitive load}

Most BMI systems are tested in quiet laboratory environments, where users are able to concentrate on the task at hand with minimal distractions. BMI users in the real world have to deal with much more complex situations, including the cognitive load of the task being performed, emotional responses, interactions with other people, and possibly even safety considerations. Careful study of the effects of cognitive load on the efficacy of BMI controls is necessary in order to determine whether BMI's could be used for rather "quiet" inhome everyday living situations up to challenging living situations of spaceships or space stations.

\section{FUTURE DIRECTIONS}

While brain-actuated robots have been demonstrated in the laboratory, this technology is not yet ready to be taken out and used in real-world situations. A critical issue is how to improve the robustness of BMIs with the goal of making them more practical and reliable. Several issues can play a major role to this end:

- Online adaptation of the interface to the user to keep the BCI constantly tuned to its owner [17].

- Analysis of neural correlates of highlevel cognitive and affective states (errors, alarms, attention, frustration, confusion...). Information about these states is embedded in the EEG together with the mental commands intentionally generated by the user [16].

- Monitoring electrical activity all across the brain with high spatial accuracy (monitor local neuron activities) without implanting electrodes but rather by a noninvasive estimation from scalp signals [18].

- Nature and role of feedback for brainactuated control, and in particular the use of multiple modalities of feedback to accelerate user training and facilitate accurate control of the robots.

- Improvement of the recording technology, that currently requires the use of gel to improve the conductivity of the electrical signals generated in the brain.

\section{CONCLUSIONS}

This paper suggests the use of noninvasive Brain Machine Interfaces for space applications. In our opinion, the introduction of novel interfaces capable to read and interpret brain activities to control external artificial systems is expected to happen in the relatively near future. So far, this technology has not however reached the readiness level necessary for any realistic implementation. Deep investigations and further developments are therefore expected.

Among the main advantages that Brain Machine Interfaces could bring to space activities we emphasise the possibility of reducing the need for extra-vehicular activity, reducing control input delays, allowing multi-task operations, improving interfaces with artificial intelligent systems during intra-vehicular activities and allowing augmentation of astronaut operation capabilities.

We believe that Brain Machine Interfaces based on the measurement of electroencephalographic signals are the most promising for being introduced in the space sector in the near term, mainly because of the portability of the interface. 
However, we do not exclude that other technologies, the use of which is now limited by the size and mass of their signal acquisition equipments (e.g. MEG, NIRS, fMRI), could be used in the future.

\section{REFERENCES}

[1] Tonet O., Tecchio, F., Sepulveda, F., Citi, L., Tombini, M., Marinelli, M., Focacci, F., Laschi, C., Dario, P., Rossini, P.M. (2006) Critical Review and Future Perspectives of Non-Invasive BrainMachine Interfaces, ESA Ariadna Study 05/6402, ESA Contract 19707/06/NL/HE.

[2] Carpi, F., De Rossi, D. (2006) Non Invasive Brain-Machine Interfaces, ESA Ariadna Study 05/6402, ESA Contract 19706/06/NL/HE

[3] Millán, J. del R., Ferrez, P., Buttfield, A. (2006) Non Invasive Brain-Machine Interfaces, ESA Ariadna Study 05/6402, ESA Contract 19708/06/NL/HE

[4] Carmena, J.M., Lebedev, M.A., Crist, R.E., O’Doherty, J.E., Santucci, D.M., Dimitrov, D.F., Patil, P.G., Henriquez, C.S., Nicolelis, M.A. (2003) Learning to control a brain-machine interface for reaching and grasping by primates. PlosBiology, Vol 1 (2), pp.193-208

[5] Hochberg, L.R.,Serruya, M.D., Friehs, G.M., Mukand, J.A., Saleh, M. Caplan, A.H., Branner, A., Chen, D. Penn, R.D., and Donoghue, J.P. (2006). Neuronal ensemble control of prosthetic devices by a human with tetraplegia. Nature 442, 164-171.

[6] Pfurtscheller, G. \& Neuper, C. (1997). Motor Imagery Activates Primary Sensorimotor Area in Humans. Neurosci. Lett. 239, 65-68.
[7] Wolpaw, J. R., Birbaumer, N., McFarland, D. J., Pfurtscheller, G., Vaughan, T. M. (2002) Brain-Computer Interfaces for Communication and Control, Clinical Neurophysiology 113, pp.767-791

[8] Millán, J.d.R., Renkens, F., Mouriño, J. \& Gerstner, W. (2004). Non-Invasive Brain-Actuated Control of a Mobile Robot by Human EEG. IEEE Trans. Biomed. Eng. 51, 1026-1033.

[9] Farwell, L.A. \& Donchin, E. (1988) Talking Off the Top of Your Head: Toward a Mental Prosthesis Utilizing Event Related Brain Potentials, Electroenceph. Clin. Neurophysiol. 70, 510-523.

[10] Millán, J. del R., Renkens, F., Mouriño, J., Gerstner, W. (2004). BrainActuated Interaction. Artif. Intell. 159, pp. 241-259.

[11] Birbaumer, N., Ghanayim, N., Hinterberger, T., Iversen, I., Kotchoubey, B., Kübler, A., Perelmouter, J., Taub, E. \& Flor, H. (1999). A Spelling Device for the Paralysed. Nature 398, pp. 297-298.

[12] Sheridan, T.B. (1992). Telerobotics, Automation and Human Supervisory Control. MIT Press, Cambridge, Massachusetts.

[13] Lujan, B.F. \& White, R.J. (1995). Human Physiology in Space, National Aeronautics and Space Administration Headquarters.

[14] Pinsky, L.S., Osborne, W.Z., Bailey, J.V., Benson, R.E. Thompson, L.F. (1974). Light flashes observed by astronauts on Apollo 11 through Apollo 17. Science 183, pp 957-959. 
[15] Moore, M.M. (2003) Real-world applications for brain-computer interface technology, IEEE Trans Neural Syst Rehabil Eng 11(2), pp 162-165.

[16] Ferrez, P.W. \& Millán, J.d.R. (2005). You Are Wrong!-Automatic Detection of Interaction Errors from Brain Waves. In Proc. 19th Int. Joint Conf. Artificial Intelligenc.

[17] Buttfield, A., Ferrez, P.W. \& Millán, J.d.R. (2006). Towards a Robust BCI: Error Recognition and Online Learning. IEEE Trans. Neural Sys. Rehab. Eng. 14, pp. 164-168.

[18] Grave de Peralta, R., Gonzalez Andino, S., Perez, L., Ferrez, P.W., \& Millán, J.d.R. (2005). Non-Invasive Estimation of Local Field Potentials for Neuroprosthesis Control. Cogn. Process. 6, pp. 59-64. 